\title{
7
}

\section{Optimization of Curing Cycles for Thick-wall Products of the Polymeric Composite Materials}

\author{
Oleg Dmitriev and Sergey Mischenko \\ Tambov State Technical University \\ Russia
}

\section{Introduction}

Production of composites is a complex, energy-intensive, relatively time-consuming chemical-technological process. Therefore, the issue of increasing intensity of production of composite products is largely linked to the performance of technological operations for composites, such as forming, curing, heat treatment and cooling (Balakirev et al., 1990).

The quality of the thermoset composite products is an integral part of increasing the intensity of the process their of obtaining, which is mainly determined by the properties of the cured material, its macro-and microstructure and geometrical parameters of the finished product. Dependence of the quality of products from the processing parameters is determined mainly by the curing cycle, temperature - conversion of homogeneity, achieved the degree of cure, the degree of thermal degradation of the resin, as well as residual stresses.

The process of curing of thermosetting products of composites accompanied, as a rule, exothermic reaction. Due to the low thermal conductivity of the composites during their curing a significant heterogeneity of the temperature-conversion field perpendicular to layers of prepregs occurs and increases with the thickness of the product. If not optimal temperature-time cycle, this leads to significant overheating of internal layers of material products, the deviation curing degree of polymeric resin from optimal, the destruction of resin, the accumulation of internal stresses that cause the reduction of strength properties of the material, warping of finished product, etc. To remove these deficiencies and thereby improve the product quality indicators can be achieved through optimal assignment of temperature-time cycle of $U(t)$ on the surface of the product (Balakirev et al., 1990).

The principal terms of the optimal technology curing thermoset composites, which provide high quality products on the physical and mechanical properties and greater stability of these properties with respect to time, are as follows (Wu \& Joseph, 1990):

- $\quad$ products should be cured on a strictly defined temperature-time cycle;

- compaction pressure should provide the desired resin content and density of the material in the product, as well as of a given thickness;

- $\quad$ in the process of molding products should be provided with the necessary degree of curing resin in the composite;

- product after compaction shall not contain residual stresses greater than the number corresponding to the dynamic equilibrium in the range of operating temperatures and ensures greater stability over time dimensions and mechanical properties of products. 
The main parameters of the curing process of composite products are: temperature-time cycle, compaction pressure, the time of its application and duration of the process (Balakirev et al., 1990). Properties of cured polymer composite material depend strongly on the correct choice of these parameters and, consequently, the character of the curing process in time, especially at the initial stage, which lays the desired structure. Therefore, the problem of determining optimal curing cycle of composites is an important and responsible.

The main tasks that must be addressed in the selection of optimal curing of composite, that guaranteed high quality and low unit cost, are (Rosenberg \& Enikolopyan, 1978):

- reducing the temperature-conversion of inhomogeneities in the composite;

- $\quad$ reducing the duration of the curing cycle and energy consumption;

- complete curing resin in prepregs;

- compacting of the composite up to the desired thickness of the product.

Until recently, for the choice of curing cycles used empirical methods and curing cycles selected according to the results of lengthy experiments. For example, the authors of works (Rosenberg \& Enikolopyan, 1978) selected curing cycles on the basis of the conditions for obtaining maximum strength, finding the dependence of breaking stress in the bending strength of the temperature or pressure. In (Kulichikhin \& Astakhov, 1991) curing cycles of products was determined by rheological and dielectric measurements. The author of (Botelho et al., 2001) hold time during the curing thermosetting resins determined by change the viscoelastic properties. In (Stern, 1992), (Aleksashin \& Antyufeyev, 2005) curing cycle of products based on epoxy and phenol-formaldehyde epoxy resin was determined by time gelation studied using differential thermal analysis and differential scanning calorimetry. A significant shortcoming of these and many other methods is the need to use samples of small diameter and small thickness, the inability to exert the necessary pressure molding, the absence of rheological characteristics of polymeric binder, etc. Therefore, the results obtained using these methods do not fully correspond to the processes proceeding during cure of materials in real constructions.

The disadvantage of the methods of curing cycles choice is that they basically can be applied to thin-walled products (thickness 1-3 mm), which is implemented a uniform setting of cure on thickness of material, and absolutely can not be applied to thick-walled products (more than $3-5 \mathrm{~mm}$ ), in which the entire course of the curing process is determined by speed of withdrawal of heat (by thermal conductivity) to be allocated in the exothermic reaction of curing. The curing cycles of thick-walled products usually obtained on base of the curing cycles of thin plates that was obtained empirically. In doing so, the temperature of isothermal hold did not change, and hold time in the press were set to form a linear dependence on the thickness of the product (Dedyukhin \& Stavrov, 1976). This method is built without taking into account heat transfer, curing kinetics and processes that related to changes viscosity of resin, which leads to large errors.

The use for this purpose of simple calculation methods, based on the theory of similarity, allowing extrapolate the curing process parameters, found in the same conditions to other conditions of curing. For example, in (Dedyukhin \& Stavrov, 1976) obtained a formula for calculation, allowing determine of the hold time of the cure in one-step cycle, depending on temperature molds, taking into account the heating rate and activation energy of resin. However, these formulas do not contain connection the temperature curing cycle and the mechanical characteristics of the material (residual stresses) and do not take into account the possible overheating of internal layers of the product as a result of exothermic effect. Nevertheless, they used to calculate the curing cycles of thick-walled products. 
Thus, an analysis of previously used empirical and simple calculation methods revealed the following deficiencies:

a. to determine of the curing cycles of composite required a broad program of experiments;

b. the curing cycles, found to be satisfactory for this material in some conditions, may not be appropriate in other conditions, in particular, when need other thickness of material;

c. these techniques do not guarantee complete and high-quality curing of material by the selected temperature-time curing cycle.

The most complete problem of selection of curing cycle products can be solved through the combined use of optimization methods with the use of mathematical models of thermokinetic curing process and define of mathematical model parameters. This method consists in setting and solving the problem of minimizing a certain criterion of optimality (Afanasyev, 1985). It can help to determine the curing cycle of composite products for any geometrical sizes and shapes.

The first attempts for solving the problem of finding optimal curing of thick-walled products cycle of thermosetting composites were made by the authors of works (Wu \& Joseph, 1990), (Frank et al., 1991) and further developed in (Balakirev et al., 1990), (Dmitriev et al., 2009). As a mathematical model in most of these works was used a simplified system of linear differential equations of heat conduction and kinetics without the mass transfer, which limited its use and accuracy of solutions. Strict theory of solutions of such problems are currently not, therefore, in these works have used the approximate methods.

Thus, one can conclude that if there is a large diversity of methods for selecting the curing cycles the majority of them were built on the basis of empirical approaches. In a small number of mathematical methods was used the simplified set of optimization problems, based on a simple linear mathematical models, without taking into account the many phenomena of the process, for example, currents of resin under the pressure, dependence of thermophysical properties on temperature, degree of curing and filling ratio (fraction factor). In addition, almost entirely absent in the tasks of optimizing the recording of pressure forming, determining its magnitude and duration of application.

The aim is to head the prospects of using the method for calculating the optimal curing cycles of large-scale products of polymer composite materials based on mathematical modeling and numerical search of the temperature-time cycle, ensuring the creation of highquality finished product in the minimal possible time, with minimal power consumption, or with minimal residual stresses.

\section{Mathematical model and identification of its parameters}

In the mathematical description of any phenomenon or process, we solve two conflicting objectives. On the one hand it is necessary to obtain a simple model with a small number of parameters and we have research equipment for determine it. On the other hand the model should be adequate to the real process with acceptable accuracy. Use for this purpose of simple linear mathematical models without taking into account the many phenomena of the process, such as, for example, outflow the resin under the pressure, dependence of thermophysical properties on temperature, degree of cure and the filling factor leads to large modeling errors and uncertainty of the solutions. 
Technologies for obtaining large-size thick-walled products have the greatest interest. Examples of such technologies include the hot pressing in mold, autoclave vacuum forming and winding on the mandrel (Balakirev et al., 1990). Products that produced by these technologies tend to have a flat shape or a small curvature, which allows to consider such products as the unlimited plate and to use one-dimensional mathematical model of the process, although there are no obstacles to the use of more complex models. A mathematical model for each technology has its own characteristics, but at the same time, a unified framework (Dmitriev \& Shapovalov, 2001). Typically, a mathematical model of a hot curing of composites when regulating the temperature of the forming tooling $U(t)$ is a system of differential equations of heat and mass transfer, kinetics of curing, leaking (extrusion) of resin, compacting the material in the process of forming and rheological equations of state. As an example, the method of calculating the optimum curing cycles of composites for the thick-walled products or panels, produced by autoclave vacuum molding is considered.

For the autoclave vacuum molding the dominant processes in the material are heat-mass transfer and curing. A mathematical model of the autoclave molding and curing of products made of thermosetting composites in multi-layer $i=1, \ldots 5$ technology package, taking into account the outflow of resin is a system of differential equations of the following:

- heat-mass transfer:

$$
\begin{gathered}
C_{i}\left(\frac{\partial T}{\partial t}+\gamma_{i} w_{x_{i}} \frac{\partial T}{\partial x}\right)=\frac{\partial}{\partial x}\left(\lambda_{i} \frac{\partial T}{\partial x}\right)+\gamma_{i} Q_{f_{i}} \frac{\partial \beta_{i}}{\partial t}, \\
T \equiv T(x, t), L_{i-1} \leq x<L_{i}, L_{0}=0,0<t \leq t_{f}, \quad i=1, \ldots 5, \\
C_{i} \equiv C_{i}(T), \lambda_{i} \equiv \lambda_{i}(T), w_{x i}=0, Q_{f i}=0, L_{i} \equiv \text { const }, \text { at } i=1,4,5, \\
C_{2} \equiv C_{2}\left(T, \beta_{2}, \gamma_{2}\right), \lambda_{2} \equiv \lambda_{2}\left(T, \beta_{2}, \gamma_{2}\right), w_{x 2} \neq 0, Q_{f 2} \neq 0, L_{1} \leq x<L_{2}(t), i=2, \\
C_{3} \equiv C_{3}\left(T, \beta_{3}, \gamma_{3}\right), \lambda_{3} \equiv \lambda_{3}\left(T, \beta_{3}, \gamma_{3}\right), \quad w_{x 3} \neq 0, Q_{f 3} \neq 0, L_{2}(t) \leq x<l_{b l}(t), i=3, \\
C_{3} \equiv C_{3}(T), \lambda_{3} \equiv \lambda_{3}(T), w_{x 3}=0, Q_{f 3}=0, l_{b l}(t) \leq x<L_{3}, i=3,
\end{gathered}
$$

with boundary conditions: $\quad T(x, 0)=f(x), \quad 0 \leq x \leq L_{5}$,

$$
\begin{gathered}
-\left.\lambda_{1} \frac{\partial T}{\partial x}\right|_{x=0}=\alpha\left[T_{\mathrm{a}}(t)-T(0, t)\right], \quad 0<t \leq t_{f}, \\
-\left.\lambda_{5} \frac{\partial T}{\partial x}\right|_{x=L_{5}}=\alpha\left[T_{\mathrm{a}}(t)-T\left(L_{5}, t\right)\right], \quad 0<t \leq t_{f}, \\
\left.\lambda_{i} \frac{\partial T}{\partial x}\right|_{x=L_{i}-0}=\left.\lambda_{i+1} \frac{\partial T}{\partial x}\right|_{x=L_{i}+0}, \quad 0<t \leq t_{f}, \quad i=1, \ldots 4, \\
\left.T(x, t)\right|_{x=L_{i}-0}=\left.T(x, t)\right|_{x=L_{i}+0}, \quad 0<t \leq t_{f}, \quad i=1, \ldots 4,
\end{gathered}
$$

control parameters

$$
U(t)=T\left(L_{1}, t\right) \text { or } U(t)=T_{\mathrm{a}}(t),
$$


- $\quad$ kinetics of cure

$$
\begin{gathered}
\frac{\partial \beta_{i}}{\partial t}=\left\{\begin{array}{r}
\varphi_{i}(\beta) \exp \left[-E_{i}(\beta) / R T\right], \quad \beta<1, \\
0, \quad \beta=1,
\end{array}\right. \\
\beta_{i} \equiv \beta_{i}(x, t), \quad L_{i-1} \leq x<L_{i}, \quad 0<t \leq t_{f}, \\
\beta_{i}(x, 0)=\beta_{0 i}(x) \geq 0, \quad i=2,3,
\end{gathered}
$$

- outflow resin and compacting material

$$
\begin{aligned}
& w_{x_{2}}(t)=\left\{\begin{array}{cc}
\frac{d L_{r e}(t)}{d t}, & L_{2}(t)-\ell_{f l}(t) \leq x \leq L_{2}(t), \\
0, & 0<x<L_{2}(t)-\ell_{f l}(t),
\end{array}\right. \\
& w_{x_{3}}(t)=\left\{\begin{array}{cr}
\frac{d \ell_{r e}(t)}{d t}, & L_{2}(t) \leq x \leq L_{2}(t)+\ell_{b l}(t) ， \\
0, & L_{2}(t)+\ell_{b l}(t)<x<L_{3} ，
\end{array}\right. \\
& L_{2}(t)=L_{2}(0) \frac{1-\gamma_{s}}{1-\gamma_{f}}+L_{r e}(t), \quad L_{2}(0)=L_{p r \cdot \min } \frac{1-\gamma_{f}}{1-\gamma_{s}}, \\
& \frac{d L_{r e}(t)}{d t}=-\frac{P_{a}+P_{0}-\rho_{r e} g \cdot\left[\ell_{f l}(t)+\ell_{b l}(t)\right]}{\frac{1}{k_{f l}} \int_{L_{2}(t)-\ell_{f l}(t)}^{L_{2}(t)} \mu(x, t) d x+\frac{1}{k_{b l}} \int_{L_{2}(t)}^{L_{2}(t)+\ell_{b l}(t)} \mu(x, t) d x}, \quad 0<t \leq t_{f}, \\
& L_{r e}(0)=L_{2}(0) \frac{\gamma_{s}-\gamma_{f}}{1-\gamma_{f}} \\
& \mu(x, t)=\tilde{\mu}(\beta) \exp \left(\frac{E_{\mu}}{R T}\right), \quad \gamma(\mathrm{t})=\frac{\mathrm{L}_{2}(\mathrm{t})-\mathrm{L}_{2}(0)\left(1-\gamma_{\mathrm{s}}\right)}{\mathrm{L}_{2}(\mathrm{t})}, \\
& \ell_{f l}(t)=\frac{L_{2}(0)\left(1-\gamma_{s}\right)}{n}\left[1+\frac{(n-1)\left(\gamma_{s}-\gamma(t)\right)}{\gamma_{s}-\gamma_{f}}\right] \\
& \ell_{b l}(t)=\frac{L_{2}(0) \gamma_{s}-L_{r e}(t)}{\xi}, \quad \xi=V_{p o r} / V_{b l}, \\
& \gamma_{s}=\frac{\rho_{p r}(0) M_{r e}(0)}{\rho_{r e} M_{p r}(0)}, \quad \gamma_{f}=\frac{\rho_{p r \cdot \min } M_{r e \cdot \min }}{\rho_{r e} M_{p r \cdot \min }},
\end{aligned}
$$


where $C$ - volumetric heat capacity, $\mathrm{J} /\left(\mathrm{m}^{3} \cdot \mathrm{K}\right) ; E$ - activation energy of curing, $\mathrm{J} / \mathrm{mol}$; $E_{\mu}$ activation energy of viscous flow, $\mathrm{J} / \mathrm{mol} ; f$ - initial distribution of temperature, $\mathrm{K} ; Q_{f}$ - full heat of the reaction, $\mathrm{J} / \mathrm{m}^{3} ; k$ - permeability, $\mathrm{m}^{2} ; L$ - thickness of the product, $\mathrm{m} ; \ell_{\mathrm{bl}}$ - the depth of penetration of the resin in the bleeder, $\mathrm{m}$; $\ell_{f l}$ - the thickness of the tightly compressed layers of filler, $\mathrm{m} ; M$ - mass, $\mathrm{kg} ; n$ - number of layers of prepreg in the product; $P_{0}, P_{a}$ - the pressure in the autoclave and vacuum bag, Pa.; $R$ - universal gas constant, $\mathrm{J} /(\mathrm{mol} \cdot \mathrm{K}) ; \mathrm{T}$ - temperature, $\mathrm{K} ; \mathrm{T}_{a}$ - the temperature of heating air, $\mathrm{K} ; t$ - time, $\mathrm{s} ; x$ - spatial coordinate, $\mathrm{m} ; V$ - volume, $\mathrm{m}^{3} ; w$ - speed of the current resin, $\mathrm{m} / \mathrm{s} ; \alpha$ - heat-removing factor, $\mathrm{W}\left(\mathrm{m}^{2} \cdot \mathrm{K}\right) ; \beta$ - the degree of curing; $\gamma$ - the mass contents of the resin in the prepreg; $\varphi$ kinetic function, $1 / \mathrm{s} ; \lambda$ - thermal conductivity, $\mathrm{W} /(\mathrm{m} \cdot \mathrm{K}) ; \mu$ - dynamic viscosity of the resin, Pa.s; $\rho$ - density, $\mathrm{kg} / \mathrm{m}^{3} ; \xi$ - porosity bleeder;

indexes: re - resin; fl - fabric filler; bl - bleeder; $\mathrm{pr}$ - prepreg; $\mathrm{s}$ - start; $\mathrm{f}$ - final; $\mathrm{a}$ - the air.

Moreover, $x=0$ corresponds to the lower surface of the metal tool plate, $x=L_{5}$ - corresponds to the upper surface of the airweave breather layer; $i=1,2, \ldots 5$ - correspond to each layer of a technological package: $i=1$ - metal tool plate, $i=2$ - laminate prepreg, $i=3$ - bleeder, $i=4$ perforated metal sheet, $i=5$ - airweave breather layer.

In autoclave vacuum molding the temperature of heating air $T_{\mathrm{a}}(t)$ or the temperature of the forming tooling $T\left(L_{1}, t\right)$ are control parameters $U(t)$.

The proposed model describes only the changes in the size of the composite products, which are caused by changes in the content of the resin. Shrinkage caused by changes of molecular structure of the resin during curing is not considered.

\begin{tabular}{|l|l|c|}
\hline \multicolumn{1}{|c|}{ № } & \multicolumn{1}{|c|}{ Determined experimentally in the CMS } & Designation \\
\hline \multicolumn{3}{|c|}{} \\
\hline 1 & Volumetric heat capacity & $C(T, \beta, \gamma)$ \\
\hline 2 & Thermal conductivity & $\lambda(T, \beta, \gamma)$ \\
\hline 3 & Full heat of the reaction & $Q_{f}$ \\
\hline 4 & Activation energy of curing & $E(\beta)$ \\
\hline 5 & Kinetic function of curing & $\varphi(\beta)$ \\
\hline 6 & Activation energy of viscous flow & $E_{\mu}$ \\
\hline 7 & Dynamic viscosity of the resin during cure & $\widetilde{\mu}(\beta)$ \\
\hline 8 & Permeability of filler & $k_{f l}$ \\
\hline 9 & Permeability of bleeder & $k_{b l}$ \\
\hline \multicolumn{2}{|c|}{ Determine additional } & $\xi$ \\
\hline 10 & Porosity of bleeder & $L_{p r . m i n}$ \\
\hline 11 & Thickness of the tightly compressed layers of filler & $L_{o}$ \\
\hline 12 & Initial thickness of the product & $\beta_{o}$ \\
\hline 13 & Initial prepreg degree of curing & $\gamma_{s}$ \\
\hline 14 & Initial mass contents of the resin in the prepreg &
\end{tabular}

Table 1. Determined experimentally parameters of the mathematical model

The forming often occurs without the outflow of resin from the prepreg in the technological package or in a closed mold by direct hot pressing. Then the mathematical model is simplified to two differential equations, this equation of heat conduction and kinetics. Accordingly, the number needed to identify the characteristics of the material decreases. 
One of key parameters of the mathematical model of curing process are the characteristics of a material, both in the cured a state and in the uncured state, and also during curing. They are not constant, but are a function of temperature $T$, the degree of curing $\beta$, resin content $\gamma$ or time $t$, which are subject to identification by experimental data (see table 1).

Typically, these parameters are determined from experiments using special techniques and devices. For their definitions are used: differential scanning calorimeter (DSC) for studying the thermal processes occurring in the prepreg on heating, thermomechanical analyzer (TMA), dynamic mechanical analyzer (DMA), thermal conductivity meters, etc.

However, currently available experimental devices and apparatus are intended primarily to study the properties of solid materials or cured composites or curing kinetics of the small size of samples in the absence of process pressure. Devices for the determination of rheological characteristics of thermosetting resins, typically built on traditional classical rheology and can measure the characteristics of the pure resin without the structural features of composite and production process (Kulichikhin \& Astakhov, 1991). This restricts the use of standard methods and devices for studying the curing of composites, because they do not fully possible to determine all the parameters of a mathematical model of the curing process with all the relationships and features. Thus, to solve this problem it is necessary to use special methods and measuring devices that allow to determine the characteristics of composites in conditions close to conditions for the production of composites in the process. This path will allow more finely into account all the circumstances connected with the study of properties of composites and to obtain more precise parameters of the mathematical model.

Characteristics that are determined in conditions close to technological process are in some sense effective, that correspond to the peculiarities of the process and bear in itself factors and effects that not considered at the mathematical description and simulation. So promising are the methods and experimental devices that allow to study materials for the real designs using special samples and reproduce in the study of process conditions, that are process pressure, heating rate, peculiarities laying prepreg, study filled composite to replace the study of pure resin, etc. These requirements relate to the presence of interphase interactions occurring at the interface of resin-filler and influencing the kinetics of curing, as well as the influence of process pressure and laying on the thermal conductivity of the composite. An important condition for developing such devices, along with the ability to simulate technological modes of cure, is the ability to create special boundary conditions are necessary to simplify the algorithms for processing the experimental data and the desired accuracy of determine parameters.

Given the fact that the process of curing and polymer composites are highly informationintensive objects, their properties are often complicated functions, to solve the problem of determining the parameters of the mathematical model is necessary to use automation and computer equipment.

To determine the material characteristics and other parameters of the mathematical model we have developed a number of methods and the computer-measuring system (CMS) research of the curing of composites (Dmitriev et al., 2010). CMS allows to explore in the process of curing all the characteristics needed to solve the equations of the mathematical model of curing composites (1)-(10): thermophysical characteristics of composites $C(T, \beta, \gamma)$, $\lambda(T, \beta, \gamma)$ depending on the temperature $T$, the degree of curing $\beta$ and resin content $\gamma$, the rate of evolved heat of cure reaction $W(t)$, full heat of the reaction $Q_{f}$, kinetic characteristics, that 
are activation energy of curing $E(\beta)$, the kinetic function $\varphi(\beta)$, which includes the speed and order of chemical reactions, rheological characteristics, that are activation energy of viscous flow $E_{\mu}$ and effective viscosity of resin $\tilde{\mu}(\beta)$ in the curing. Permeability fiber filler $k$ is determined by CMS in conjunction with the effective viscosity as a complex $\tilde{\mu}(\beta) / k$ and does not require additional study, but if necessary it can be determined with the help of special equipment. Other parameters of the material and products, such as the density $\rho$, the thickness of the product $L$, mass $M$, the number of layers of prepreg in the product $n$ is determined by standard methods or set.

The research composites by CMS produced in the measuring cell in the range of temperatures from room to $270{ }^{\circ} \mathrm{C}$.

The object of the experimental study is a specially prepared sample, which is a package thickness of $5 \ldots 20 \mathrm{~mm}$, assembled from several sections of the researched prepregs cut to a square with side $100 \mathrm{~mm}$. Stacking layers of prepregs and orientation fiber of the sample performed similarly to industrial products: unidirectional, cross or cross-bias, etc. Samples of materials prepared on these conditions and their size allows to take into account in studied effective characteristics the rheological processes taking place during curing, the especial of stacking layers of prepreg in the product, to average a local inhomogeneity of the structure of materials and factor content of resin, which does not allow to perform other standard methods and devices, including scanning calorimetry method.

By means CMS in the measuring cell is organized the process of heating the studied sample, described by the (1) - (10), and measured over time following values: the time $t$, voltage of the heater $u(t)$, the temperature in the $2 \ldots 5$ points on the thickness of the sample $T(x, t)$, the thickness sample $L(t)$, the force pressing the sample $F(t)$, dielectric permittivity $\varepsilon(t)$ and dielectric losses $\tan \delta(t)$.

Research carried out for each sample in two phases: heating to cure and re-heating after cooling. During the first phase studied the kinetics of the cure, the flux of resin and the effective thermophysical characteristics of $C_{w}(t(T)), \lambda_{w}(t(T))$, including the rate of evolved heat of cure reaction $W(t)$. In the second phase studied the properties of cured material $C_{\mathrm{cd}}(t(T)), \lambda_{\mathrm{cd}}(t(T))$. On the basis of these data are calculated $C(T, \beta, \gamma)$ and $\lambda(T, \beta, \gamma)$. To research the kinetic and rheological characteristics conducted experiments on the cure at least for two samples with different rates of heating. The conditions of the experiment, ie voltage of core heater $u$, which determines the heating rate and the force $F$ on the sample, are chosen to reproduce the technology modes and to ensure the minimum error of the desired characteristics. Dielectric characteristics of $\varepsilon(t)$ and $\tan \delta(t)$ are needed to determine the moment of pressure application and the completion of curing.

In the experiment CMS performs the primary processing of data and recording it to the hard disk, as well as testing and monitoring of the system. At the end of the experiment is performed calculations researched characteristics.

As a method of determining the thermophysical characteristics of $C(T, \beta, \gamma), \lambda(T, \beta, \gamma)$ used the method of integral characteristics (Dmitriev et al., 2010). Kinetic characteristics of $E(\beta)$ and $\varphi(\beta)$ that are part of the equation of kinetics are determined by the rate of evolved heat of cure reaction $W(t)$, measured in the curing of two or more samples heated at different temperature-time modes $T_{\mathrm{av}}(t)$. By measuring the thickness $L(t)$ of curing sample at squeezing-out of resin are determined the rheological characteristics $E_{\mu}$ and $\tilde{\mu}(\beta)$. 
Calculated formulas and algorithms for processing of the experimental data obtained on the basis of established methods are the core software of CMS. Researched characteristics of composites and other parameters of the mathematical model are entered into a database of CMS and are the information basis for the automated calculation of the optimal curing cycles of the composite products for any thickness and configuration.

The results of experimental studies of the curing process and subsequent heating of the samples made of fiberglass plastic, as well as calculations of its characteristics are presented in Fig. 1-3.

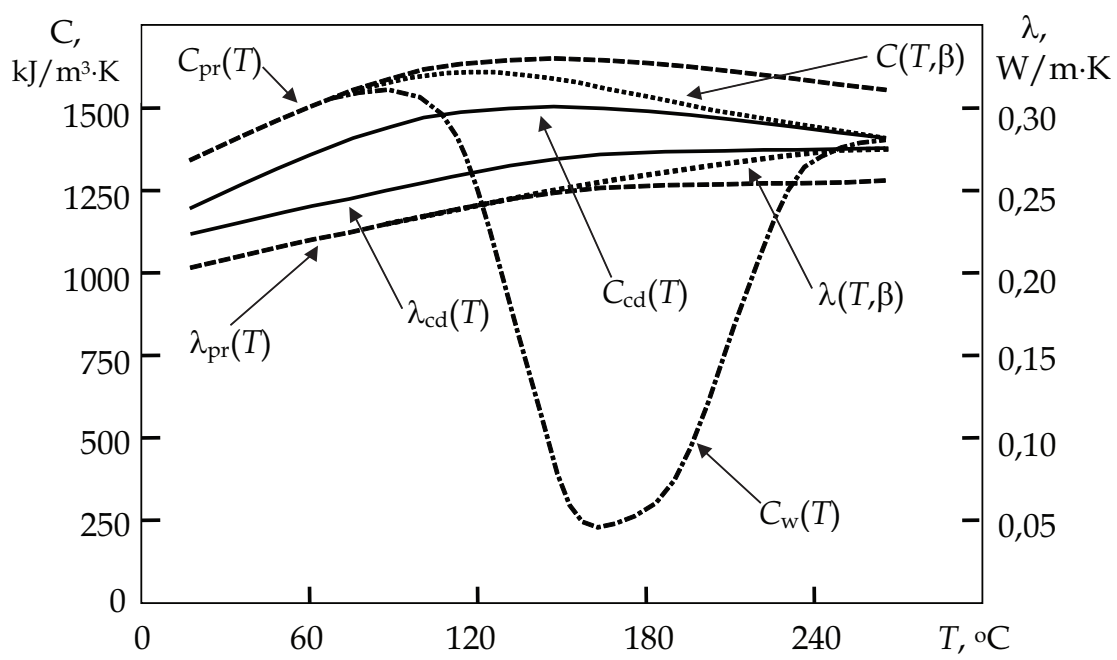

Fig. 1. Thermophysical characteristics of fiberglass plastic at $\gamma_{s}=0,51$

The initial part of effective thermophysical characteristics $C_{\mathrm{w}}(T), \lambda_{\mathrm{w}}(T)$ (Fig. 1) before the beginning of intensive curing reaction characterizes thermophysical characteristics uncured prepreg $C_{\mathrm{pr}}(T), \lambda_{\mathrm{pr}}(T)$. The beginning of intensive curing reaction (on the figure is $110 \ldots 120{ }^{\circ} \mathrm{C}$ ) corresponds to a rapid decrease in the effective volume heat capacity $C_{\mathrm{w}}(T)$, because it includes the rate of evolved heat of cure reaction $W(t)$ and it is in this part of the effective exothermic heat capacity. Effective thermophysical characteristics $C_{w}(T), \lambda_{w}(T)$ in the final stage of the experiment at a high degree of curing $\beta$ merge with thermophysical characteristics cured material $C_{\mathrm{cd}}(T), \lambda_{\mathrm{cd}}(T)$, which corresponds to the transition of composite in the cured state. As seen from Fig. 1 the dependence of thermophysical characteristics of cured composite $C_{\mathrm{cd}}(T), \lambda_{\mathrm{cd}}(T)$ and uncured prepreg $C_{\mathrm{pr}}(T), \lambda_{\mathrm{pr}}(T)$ are equidistant curves. This allows the difference between the thermophysical characteristics cured composite and uncured prepreg $\Delta C$ and $\Delta \lambda$ at the time of the beginning of the curing reaction, used for the calculation of thermophysical characteristics in the process of curing $C(T, \beta), \lambda(T, \beta)$. They are determined by thermophysical characteristics prepreg $C_{\mathrm{pr}}(T), \lambda_{\mathrm{pr}}(T)$ in the range from room temperature to the temperature of the beginning of the curing reaction, and above the temperature of the beginning of the curing reaction are determined by $C_{\mathrm{cd}}(T), \lambda_{\mathrm{cd}}(T)$ and $\Delta C, \Delta \lambda$. 


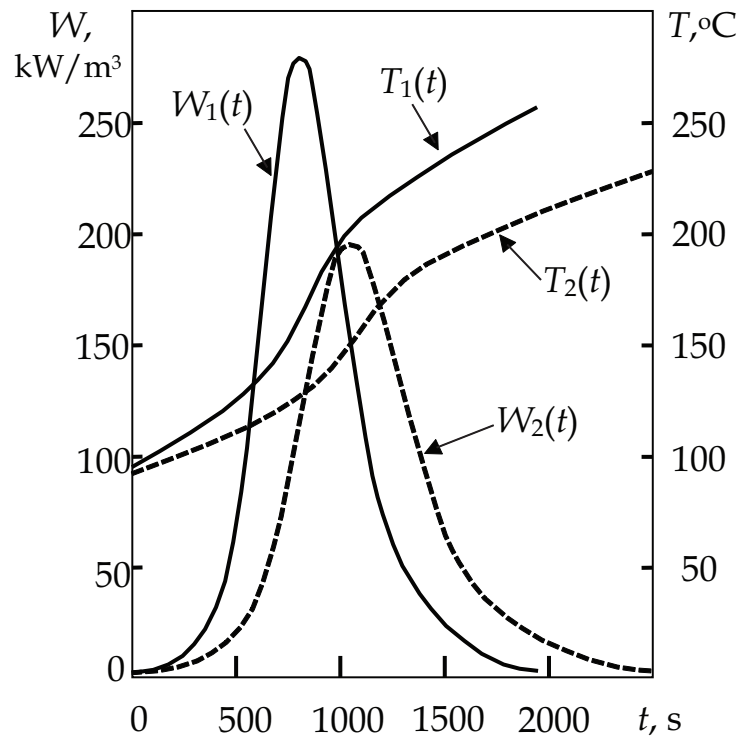

Fig. 2. Evolved heat of cure reaction of fiberglass plastic

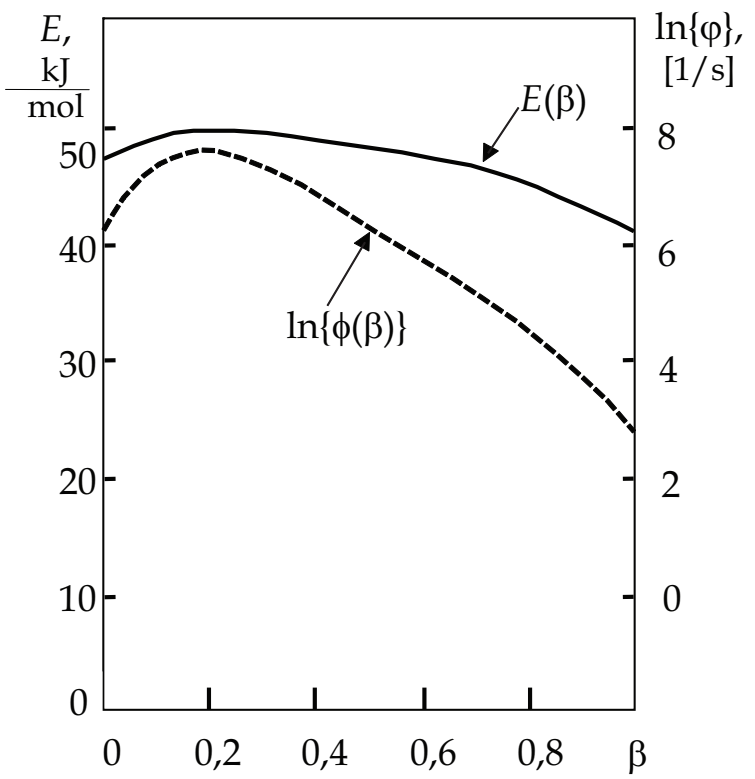

Fig. 3. Kinetic characteristics of fiberglass plastic 
The rate of evolved heat of cure reaction $W(t)$ (Fig. 2) has a pronounced extremum, corresponding to the transition from a liquid resin to the viscous-flow state (gel point). With the increase in the rate of heating the extreme of evolved heat of cure reaction $W(t)$ shifts toward higher temperatures. At the same time, the duration of heat generation and gelation time with increasing heating rate decreases. The area under the curve of the rate of evolved heat of cure reaction $W(t)$ characterizes the full heat of the reaction $Q_{f}$ of curing reaction of the resin, and the ratio of the thermal effect $Q$ to the full thermal effect of $Q_{f}$ corresponds to the degree of completeness of the process of curing or calorimetric degree of curing $\beta$.

Kinetic characteristics $E(\beta)$ and $\varphi(\beta)$ (Fig. 3) of the curing process are calculated from the rate of evolved heat of cure reaction $W(t)$, at least of two temperature-time cycles. Rheological characteristics $E_{\mu}$ and $\tilde{\mu}(\beta)$ are calculated by the change in the thickness of the samples $L(t)$ under flowing out of the resin under pressure during curing.

Thermophysical, kinetic, rheological and dielectric characteristics of composites obtained in the experiment will be automatically recorded in the database of CMS. The dielectric characteristics $\varepsilon(t)$ and $\tan \delta(t)$ is then used to calculate the correlation dependence or the function of relationship $\Psi(\beta)$ between the calorimetric and dielectric degree of cure, which is necessary for control of the manufacturing product process of composites in real time by controlling the degree of cure as well as to determine when to activate the pressure and completion of curing process (Dmitriev et al., 2010). The obtained parameters of the mathematical models are the basis for computer simulations of different situations in the curing process and calculate the optimal curing cycles of composite products of any thickness.

\section{Numerical analysis of curing cycles}

Using the once studied properties of the composite, the software of CMS allows multiple to simulate the curing process on the computer under different conditions, thus calculate the temperature-conversion field during curing of composite, as well as represent them in the form of graphs and 3D surfaces, thereby, to analyze the uneven curing of the material for the manufacture, quickly assess the advantages and disadvantages of the used curing cycle for the material and choose the right strategy to optimize the cycle.

Similar numerical studies were performed on a computer for fiberglass plastic and made a numerical analysis of the existing standard cure cycles, designed for the production of plates with thickness up to $10 \mathrm{~mm}$, we evaluated their suitability for the production of thicker plates, for example $30 \mathrm{~mm}$ thick, and we calculated the optimal curing cycles. The temperature-conversion field, ie the temperature at the surface of the plate $T_{0}$ and in the middle $T_{L / 2}$, respectively, the degree of curing $\beta$, the rate of evolved heat of cure reaction $W$ and the maximum module temperature gradient $G$ on the thickness $L$ in the process of curing fiberglass plastic on the two-step cycle thickness $L=10$ and $30 \mathrm{~mm}$ are shown in Fig. 4-5.

Based on the analysis of numerical calculations of temperature-conversion fields (Fig. 4-5) can do the following conclusions:

1. In general, the standard cycle is suitable for the manufacture of plates with thickness up to $10 \mathrm{~mm}$. Here there are small temperature gradients $G$, and drops in temperature $T_{0}-T_{L / 2}=\Delta T$, that is overheating of the inner layers due to the exothermic effect. 


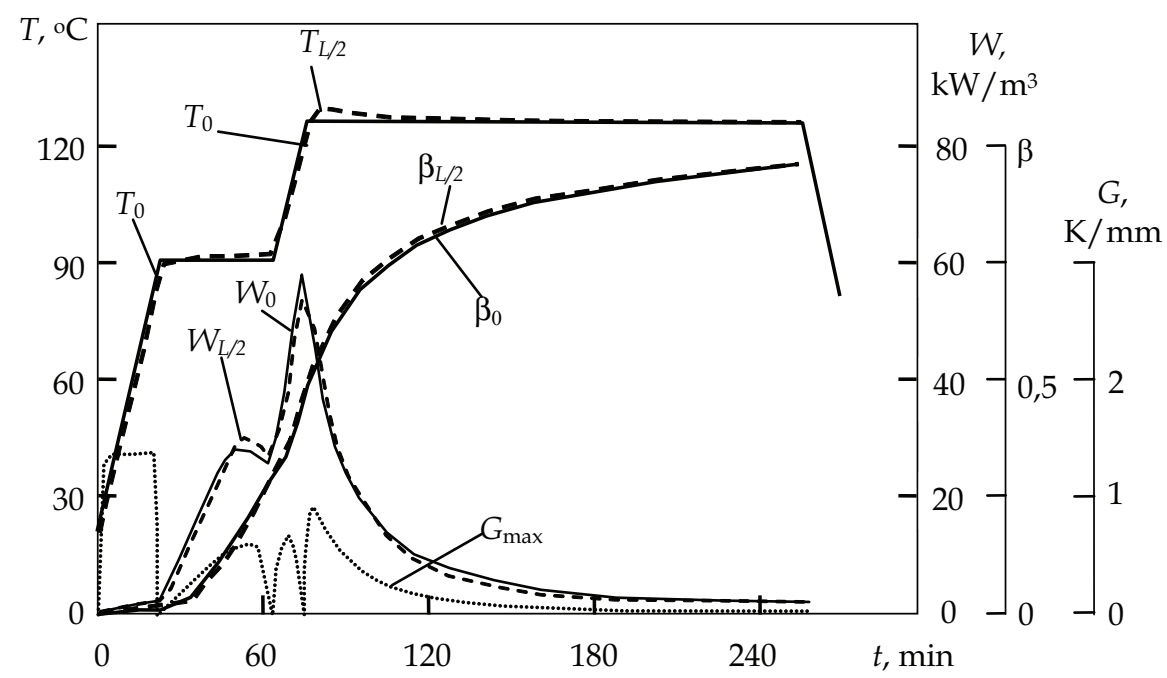

Fig. 4. Standard temperature cycle used for curing fiberglass plastic thickness $L=10 \mathrm{~mm}$

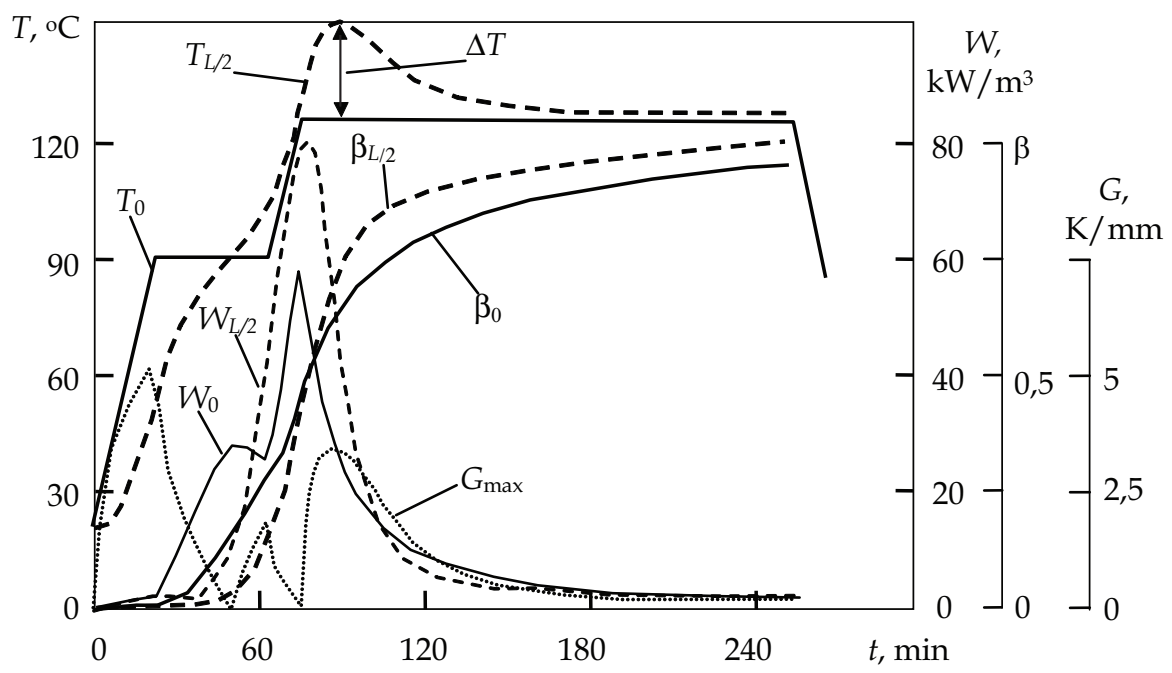

Fig. 5. Standard temperature cycle used for curing fiberglass plastic thickness $L=30 \mathrm{~mm}$

2. When curing the material thickness $L=30 \mathrm{~mm}$ at the standard cycle in the first step the temperature drops are $\Delta T_{1}=12,0 \mathrm{~K}$, the temperature gradients are $G_{1}=1,52 \mathrm{~K} / \mathrm{mm}$, in the second step, when there is formation of the structure of the material the maximum overheating $\Delta T_{2}$ and the maximum module temperature gradient $G_{2}$ reach respectively $\Delta T_{2}=25,1 \mathrm{~K}, G_{2}=2,59 \mathrm{~K} / \mathrm{mm}$. With such a temperature drop over the thickness and overheating of the inner layers of the material begin to occur the destruction processes of the resin, increasing the porosity of the composite, residual stresses accumulate. All 
this causes a decrease in the strength properties of the material, and in the manufacture of large-sized products appears to warping. Also, be aware that the large structures made of composite materials, such as elements of the wing, tail and fuselage are structures with different thickness, so the flow of polymerization processes in these zones of the composite are significantly different. In this case, the molding cycle should be chosen so as to obtain the necessary strength characteristics in most of the large area of intense construction.

These results demonstrate that the use of temperature-time curing cycle, as defined in laboratory conditions and is recommended for thin samples can not be used in the curing product of a thickness exceeding $10 \mathrm{~mm}$. In these cases, to obtain high quality products require regular technical correction cycle, or its optimization.

\section{Optimization of the curing cycles of composites}

Solution of the optimization task of the curing process of composite products is as follows: necessary to find the temperature-time cycle $U$, which is a control parameter

$$
U\left(t ; t_{f}\right), U\left(t ; Q^{*}\right), U(t ; \sigma)=\left\{T_{0}(t), T_{\mathrm{L}}(t)\right\}, \quad j=1,2, \ldots, k_{\mathrm{st}},
$$

that delivers minimum for one of the optimality criteria $I$ and provides a high quality finished product:

- $\quad$ with a minimum possible duration of curing $t_{f}$ :

$$
I_{t_{f}}=\min _{U\left(t ; t_{f}\right)} \int_{0}^{t_{f}} d \tau,
$$

- $\quad$ with minimal power consumption $Q^{*}$ :

$$
I_{Q^{*}}=\min _{U\left(t ; Q^{*}\right)} \int_{0}^{t_{f}} T_{a}(\tau) d \tau,
$$

- $\quad$ or with minimal residual stresses $\sigma$ :

$$
I_{\sigma}=\min _{U(t ; \sigma)} \int_{0}^{L}\left(\int_{\beta_{\mathrm{s}}}^{\beta_{\mathrm{f}}} p(\beta) \frac{\partial T(x, t(\beta))}{\partial x} d \beta\right)^{2} d x,
$$

in solving the equations of the mathematical model (1)-(10) or mathematical models $(\mathrm{Wu} \&$ Joseph, 1990), (Dmitriev \& Shapovalov, 2001), corresponding to the current technique of forming products

$$
\Re\left(C, \lambda, Q_{\mathrm{f}}, E_{\beta}, \varphi, E_{\mu}, \tilde{\mu}, P, \rho, k, n\right)=0, \quad j=1,2, \ldots, k_{\mathrm{st}},
$$

as well as restrictions in the form of inequalities imposed on process

$$
\max _{\substack{0 \leq x \leq L \\ t_{0}<t \leq t_{f}}} T(x, t) \leq T_{\max }, \quad \max _{\substack{0 \leq x \leq L \\ t_{0} \leq t \leq t_{f}}}\left|\frac{\partial T}{\partial x}\right| \leq \bar{\chi}, \quad \max _{t_{0} \leq t \leq t_{f}}\left\{\max _{0 \leq x \leq L} T(x, t)-\min _{0 \leq x \leq L} T(x, t)\right\} \leq \bar{\theta},
$$


taking into account the allowable for equipment temperature-time cycles

$$
U_{j_{\min }}(t) \leq U_{j}(t) \leq U_{j_{\max }}(t), \quad j=1,2, \ldots, k_{\mathrm{st}},
$$

where $p(\beta)$ - weighting function that determines the dependence of residual stresses $\sigma$ of the temperature gradient (chosen empirically); $T_{\mathrm{a}}$ - working temperature autoclave, $\mathrm{K} ; \mathrm{T}_{\max }-$ maximum allowable temperature inside the material during the curing product, $\mathrm{K} ; \bar{\chi}-$ maximum allowable temperature gradient during curing that does not cause the accumulation of domestic stresses the material, $\mathrm{K} / \mathrm{m} ; \bar{\theta}$ - the maximum allowable temperature difference on the thickness of the product that does not cause the heterogeneity of the curing material, ${ }^{\circ} \mathrm{C} ; U_{j_{\max }}(t), U_{j_{\min }}(t)$ - upper and lower limits of rate of heating the surface of the product at the $j$-th step, defined on the basis of the appropriateness of reference of the curing process, favorable conditions for the formation of curing resin structure and parameters of equipment.

Seeking a temperature-time cycle is calculated as

$$
U_{j}(t)=\left\{\begin{array}{lr}
\tilde{T}_{j-1}+\tilde{K}_{j} t, & t_{j-1}<t<t_{n_{j}} \\
\tilde{T}_{j}, & t_{n_{j}} \leq t \leq t_{j}
\end{array}, \quad j=1,2, \ldots, k_{\mathrm{st}},\right.
$$

where $\tilde{K}$ - heating rate of surface product, $\mathrm{K} / \mathrm{s} ; k_{\mathrm{st}}$ - number of steps heating; $\tilde{T}$ temperature of isothermal hold on the $j$-th step of heating, $\mathrm{K} ; t_{n_{j}}$ - moment of transition from the stage of linear heating to the isothermal hold $\tilde{T}, \mathrm{~s}$.

A condition for the finish of the $j$-th step of heating and the transition to a $j+1$ step are:

$$
\max _{\substack{0 \leq x \leq L \\ t_{j-1}<t<t_{j}}} \frac{\partial \beta_{j}}{\partial t}-\max _{0 \leq x \leq L} \frac{\partial \beta_{j}}{\partial t} \geq \eta_{j}, \max _{\substack{0 \leq x \leq L \\ t_{j-1}<t<t_{j}}} T_{j}(x, t)-\max _{0 \leq x \leq L} T_{j}(x, t) \geq \vartheta_{j}, j=1,2, \ldots, k_{\mathrm{st}} .
$$

A condition for the finish of solving the problem (11)-(19) is to achieve a given degree of cure of $\beta_{f}$ throughout the thickness of the product:

$$
\min _{\substack{0 \leq x \leq L \\ t_{0}<t<t_{f}}} \beta(x, t) \geq \beta_{f}
$$

Conditions (19) associated with the requirement of intensive curing process, so if the speed of curing decreased on the value of $\eta_{j}$ and maximum temperature on the thickness of the product reduced on the value of $\vartheta_{j}$, then execute the transition to $j+1$ step of heating and isothermal hold. Compliance with (20) guarantees the attainment by all points of the product specified value degree of finality to the process of curing $\beta_{f}$.

The main criterion for the quality of the finished product is its solidity, the lack of delaminating and buckling. Developing common criteria for monolithic reinforced composites is associated with large experimental difficulties and the lack of reliable experimental data on the study of strength and deformation properties of composites at elevated temperatures, especially in the curing process. Usually the monolithic criterion is given in the form of an inequality, which limits the residual and thermal stresses within the 
transverse compressive strength and stretching. However, such an assignment criterion of quality products also requires a simulation of the stress-strain state of curing composite and, hence, the definition of a large number of temperature - and conversion - dependent anisotropic mechanical properties such as modules of the elastic, Poisson's coefficients, modules of shear, coefficients of thermal expansion, etc. Therefore, the criterion of the quality of the finished product useful to associate with a temperature-conversion parameters of cure and values of the restrictions (16), which define the relationship with the mechanical properties of the composite, to determine experimentally.

From an examination of the physical essence of the physico-chemical transformations implies that the curing process should be limited to a maximum temperature $T_{\max }$, which are beginning to take place in connection undesirable side reactions associated with the destruction of resin, resulting in deteriorating the mechanical properties of cured products. This temperature is determined by the chemical composition of resin and this $T_{\max }$ has a certain value for each resin.

In the curing process is usually part of the evolved exothermic reaction heat is transferred into the environment, so when curing product in it there is a temperature gradient in the direction of the cooling surface. The appearance of the temperature-conversion irregularities in the product leads to residual stresses that exceed the limit of long-term strength of the material, resulting in possible cracks, delaminating and violation of a monolithic product. For practical purposes, a comprehensive description of the inhomogeneity of the temperature-conversion field is a temperature gradient, which is directly related to the gradient of the degree of completion of the curing process.

Effects of temperature gradients in the physical and mechanical properties that arise in the curing on the thickness of the material studied with the help of special experiments, consisting in testing the mechanical properties of thin samples cured in the CMS in the context of an artificially-created temperature gradients. Setting limits damaging stresses in the bending was set temperature gradient $\bar{\chi}$, which for fiberglass and carbon are in the $0,2-1 \mathrm{~K} / \mathrm{mm}$.

In the curing of thick-walled products, one of the most undesirable modes is the curing in conditions of the front exothermic reaction because it is the most favorable conditions for the formation of residual stresses. To prevent the spread of the reaction front with the optimization process of cure can be limiting the maximum temperature difference on the thickness of the product. In addition, the large difference of temperatures on the thickness of the product contribute to the formation of various structures on the segments of the material and increase the residual stress. Limit values for the temperature difference on the thickness of products $\bar{\theta}$ for fiberglass plastics and carbon plastics derived from the testing of mechanical properties specially cured specimen is $5 \sim 8^{\circ} \mathrm{C}$.

The choice of optimality criterion is the most subtle and responsible step in solving the problem of optimization. Based on the needs of the production of large thick-walled products made of thermosetting polymer composites, there are three main areas in the selection objective. The first - the creation of the finished products meet certain quality criteria for the minimum possible time. The second trend - the creation of high-quality finished product with minimal power consumption. Finally, the third area concerns the creation of structures products free of residual stresses, or with minimal residual stresses in a balanced state. Depending on the purposes of this decision is selected the corresponding optimality criterion (12), (13) or (14). If necessary, can be solved also the multi-criteria task of search for optimal curing cycle. 
From the chemical and technological considerations the most reasonable are step curing cycles. Therefore, we can assume that in our case, the kind of a required temperature-time cycle (18) is already set, it is necessary to find only optimum speeds of heating of a product surfaces $\tilde{\mathrm{K}}_{j}$ on everyone $j=1,2, \ldots, k$ steps, the temperature of isothermal holds $\tilde{\mathrm{T}}_{j}$, their durations $t_{j}$ and the number of steps heating $k_{\mathrm{st}}$. As a result of solution of the optimization problem defines the optimal boundary temperature-time cycles of the curing product of composites, in which the curing time $t_{f}$ or energy $Q^{*}$ or the residual stress $\sigma$ will be minimal. Meeting the challenge of finding the best treatment based on an incremental optimization of each of the $j$-th step of heating. For the optimization of the curing cycles of composite material products promising to use the penalty function method in conjunction with the algorithm of sequential quadratic programming, which is the core system software.

On the basis of studying the characteristics of fiberglass plastic by the developed CMS, we calculated the optimal curing cycles flat products of thickness 10 and $30 \mathrm{~mm}$. The calculated optimal curing cycles plates shown in Fig. 6-7, and Table. 2.

The calculations of the optimal curing cycles performed with the following restrictions: in the first step of heating the allowable on thickness the temperature gradient is $G=1 \mathrm{~K} / \mathrm{mm}$ and permissible overheating of the inner layers of $\Delta T=8^{\circ} \mathrm{C}$, in the second and third steps of heating allowable the temperature gradient is $G=0,4 \mathrm{~K} / \mathrm{mm}$ and the permissible overheating of the inner layers of $\Delta T=5^{\circ} \mathrm{C}$ (see Fig. 6-7 and Table 2). Increased temperature gradient in the first step of heating caused by reason of reducing the duration of the first hold, because degree of cure is not reached the point of gelation and the unevenness of the temperature-conversion field does not affect the accumulation of residual stresses.

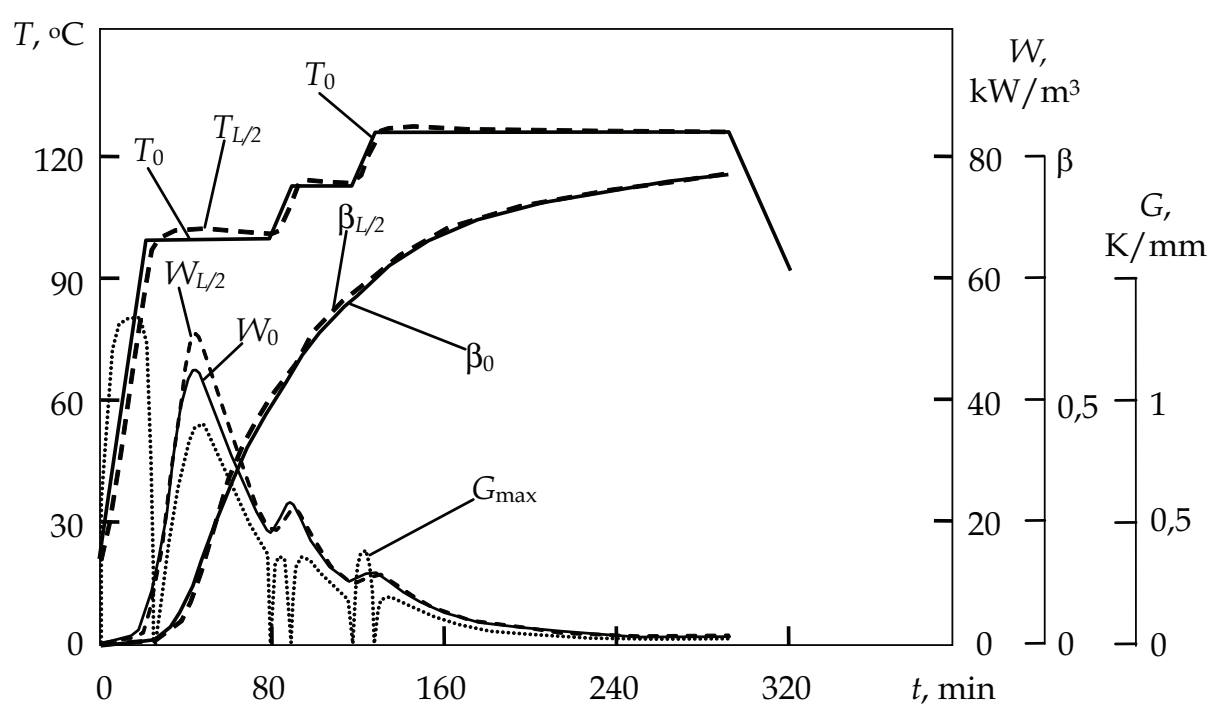

Fig. 6. Optimal temperature cycle of curing fiberglass plastic thickness $L=10 \mathrm{~mm}$

Often to removing the solvent, which is present in the resin, in the first step operates a lowtemperature hold at $80 \ldots 90{ }^{\circ} \mathrm{C}$. In this case, if the temperature is calculated by the 
optimization exceeds the specified value, then the temperature of the first hold is not selected, but rigidly defined, and its minimum duration is determined by the time required for evaporation of the solvent.

As a result of calculations, the obtained curing cycles differ from the standard cycle of smaller drops in temperature over the thickness, much smaller temperature gradients and gradual increase in the degree of curing.

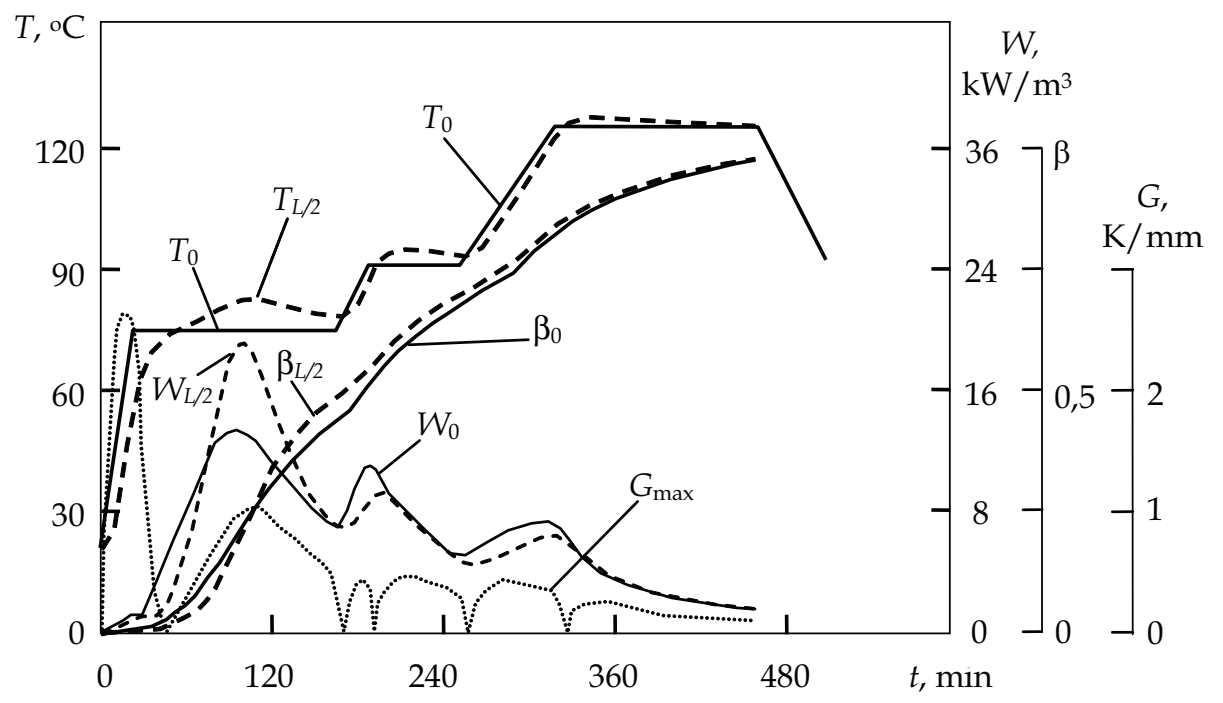

Fig. 7. Optimal temperature cycle of curing fiberglass plastic thickness $L=30 \mathrm{~mm}$

\begin{tabular}{|c|c|c|c|c|c|c|c|c|}
\hline \multirow{2}{*}{$\begin{array}{c}\text { Thickness } \\
\text { of the } \\
\text { product } \\
L, \\
\mathrm{~mm}\end{array}$} & \multirow{2}{*}{$\begin{array}{l}\text { Step } \\
\text { heating } \\
\quad j\end{array}$} & \multicolumn{7}{|c|}{ Curing cycle parameters } \\
\hline & & $\begin{array}{c}\text { Rate of } \\
\text { heating } \\
\widetilde{K}_{j} \text {, } \\
\mathrm{K} / \mathrm{min}\end{array}$ & $\begin{array}{c}\text { Heating } \\
\text { time } \\
t_{j \mathrm{~s}} \\
\text { min }\end{array}$ & $\begin{array}{c}\text { Curing } \\
\text { temperature } \\
\widetilde{T}_{j}, \\
{ }^{\circ} \mathrm{C}\end{array}$ & $\begin{array}{c}\text { Curing } \\
\text { time } \\
t_{j h} \\
\text { min }\end{array}$ & $\begin{array}{c}\text { Duration } \\
\text { of curing } \\
t_{f,} \\
\text { min }\end{array}$ & \begin{tabular}{|c|} 
Max. \\
gradient \\
$G$, \\
$\mathrm{K} / \mathrm{mm}$
\end{tabular} & $\begin{array}{c}\text { Max. } \\
\text { overheating } \\
\Delta T \text {, } \\
\mathrm{K}\end{array}$ \\
\hline \multirow{3}{*}{10} & 1 & 3,0 & 36,7 & 100,0 & 44,5 & \multirow{3}{*}{292} & 0,9 & 2,4 \\
\hline & 2 & 1,7 & 7,1 & 112,3 & 29,1 & & 0,4 & 1 \\
\hline & 3 & 1,3 & 9,7 & 125,0 & 180,3 & & 0,3 & 0,5 \\
\hline \multirow{3}{*}{30} & 1 & 2,4 & 22,5 & 74,1 & 144,0 & \multirow{3}{*}{460} & 1,0 & 7,8 \\
\hline & 2 & 0,8 & 21,7 & 90,5 & 66,8 & & 0,4 & 3,4 \\
\hline & 3 & 0,5 & 66,1 & 125,0 & 106,4 & & 0,3 & 2,0 \\
\hline
\end{tabular}

Table 2. Optimal temperature cycles of curing fiberglass plastic 
For comparison, Fig. 8 shows the existing standard cycle for $L=5 \mathrm{~mm}$, and calculated the optimal cycles for $L=10,20,30$ and $40 \mathrm{~mm}$. As can be seen from the graphs (Fig. 6-8), at the increase in thickness of the product duration of curing process significantly increases and the temperature of the first and subsequent isothermal holding markedly decreased, while the evolved heat of cure reaction in the early stages of the process also reduced. This reduces the heterogeneity of the temperature and conversion fields to the specified levels $\bar{\chi}, \bar{\theta}$ and leads to qualitative improvements of products. Due to reduction of the isothermal holding temperature levels and increase their duration reduced the intensity of the process, and the total duration of isothermal holding for thick-walled products increases. With increasing thickness of the product increases the number of steps heating $k_{\mathrm{st}}$, which is associated with the desire of optimization algorithm to intensify the curing process while satisfying the restrictions (16). These curing cycles are optimal in terms of strength characteristics and minimal residual stress, ensure high quality and low cost products due to the minimum duration and power consumption. They had no sudden changes in temperature and degree of cure in thickness, which contributes to the formation of relaxed homogeneous structure of the composite.

Similar properties have been studied and calculated the optimal curing cycles flat products in thicknesses from 5 to $50 \mathrm{~mm}$ made of several types of materials such as fiberglass plastics, organic plastics, carbon fiber reinforced plastics and rubbers. As an example, Fig. 9 also shows the temperature-time cycles of curing carbon fiber reinforced plastic thickness to 30 $\mathrm{mm}$.

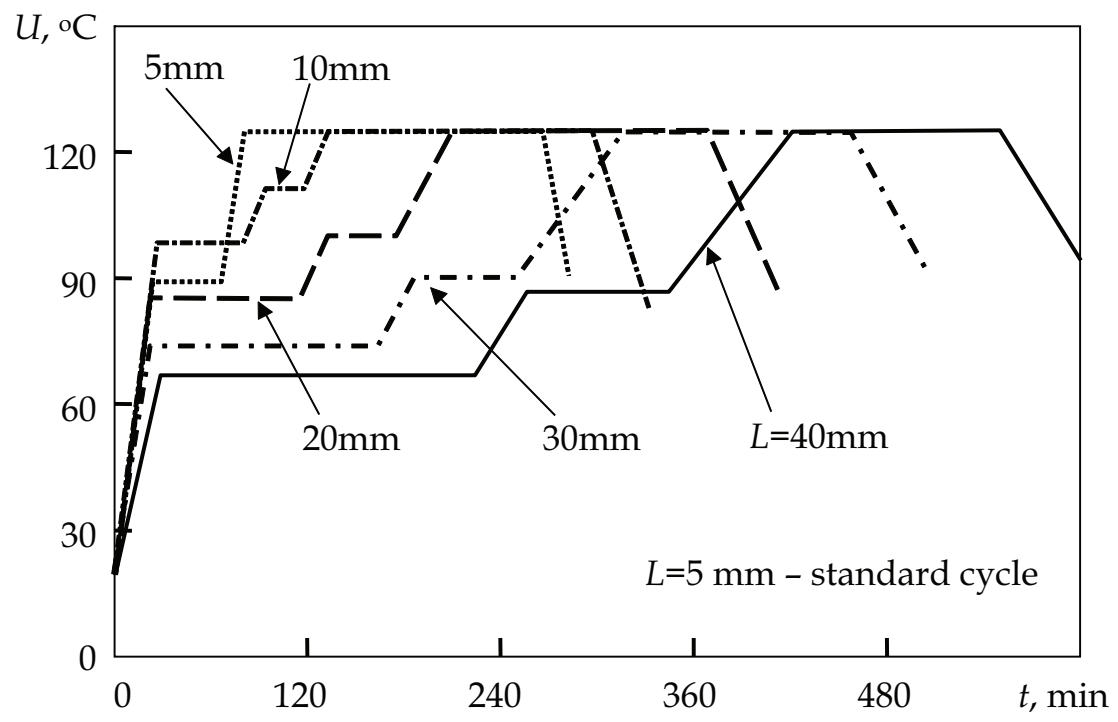

Fig. 8. Optimal cure cycles of flat products of various thickness $L$ of fiberglass plastic

The criterion for the end of the curing process is to achieve of dielectric permittivity to ended maximum allowable values and stabilize the dielectric loss.

The analysis and comparison of the calculated curing cycles flat products made of composites with existing cycles designed for thin products demonstrated the feasibility of 
widespread use of the method of mathematical modeling and optimization for testing of existing and calculation of the curing cycles of new composite materials, as well as the further development of the method.

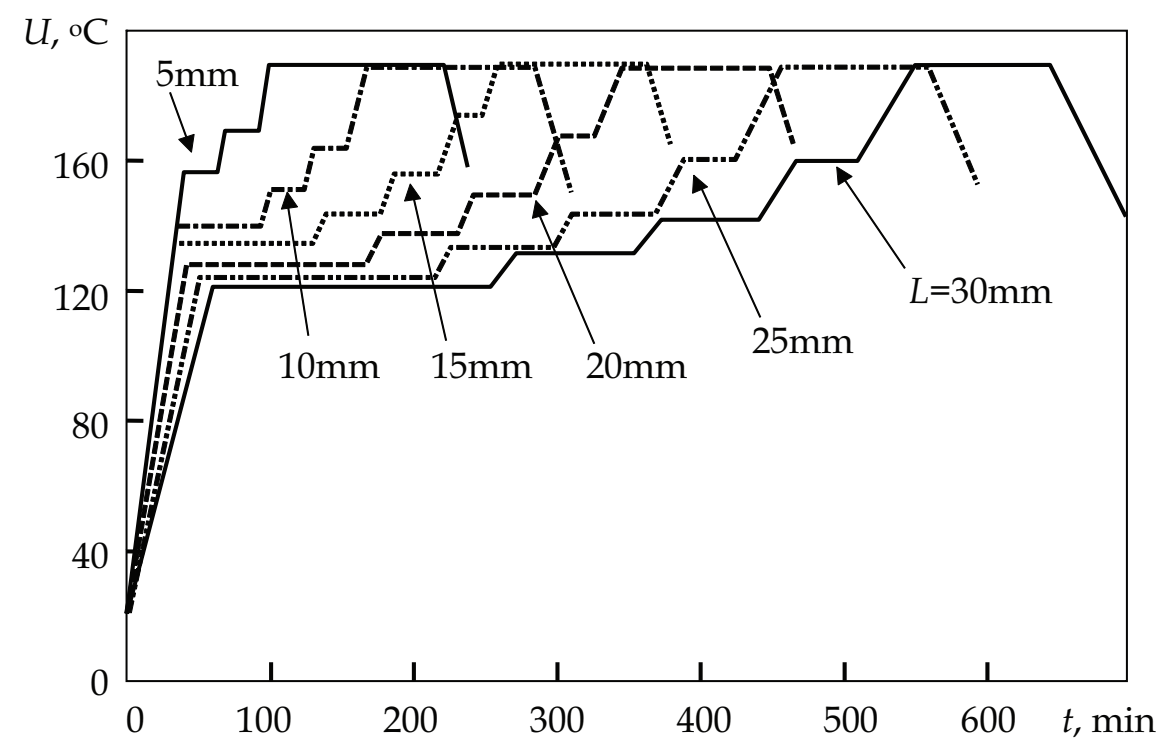

Fig. 9. Optimal cure cycles of flat products of various thickness $L$ of carbon plastic

\section{Conclusion}

Presented an example of determining the optimal curing cycles of plates of different thickness of the composites based on thermosetting resins demonstrates the need to implement in practice when determining the curing cycles of the method of mathematical simulation and optimization. The method allows to repeatedly under different conditions to simulate the curing process on the computer, that is calculate the temperature-conversion field during curing of composites, as well as represent them in the form of graphs and 3D surfaces, thereby analyze uneven curing and quickly assess the advantages and disadvantages of the curing cycle for the material and choose the correct strategy for optimizing the production cycle, thus obtain high quality finished products with minimal energy consumption, or with minimal residual stresses.

\section{References}

Afanasyev, Y.A (1985) The criterion of optimization in optimal control of technological processes of heat treatment of products made of composite polymer materials. Mehanika kompozitnyh materialov, No. 6 (December 1985) pp. 1066-1073

Aleksashin, V.M. \& Antyufeyev, N.V. (2005) Application of thermal analysis to research the adhesive compositions. Klei. Germetiki. Tekhnologii, No. 12 (December 2005) pp. 2831 
Balakirev, V. S., et al. (1990). Automated Production of Composite Material Items, Khimiya, Moscow

Botelho, E.C., Rezende, M.C. \& Scherbakoff, N. (2001) Rheological Studies Applied in the Processing and Characterization of Carbon/Carbon Composites. Journal of Composite Materials, Vol. 4, (2001) pp. 359-369.

Dedyukhin, V.G. \& Stavrov, V.P. (1976) Pressed fiberglass plastics, Khimiya, Moscow

Dmitriev, O.S. \& Shapovalov, A.V. (2001) Mathematical modeling of curing products of polymer composite materials in an autoclave by vacuum forming in a multilayered technological package, Proc. of the Intern. Conf. on Theory and Practice of Technology of Article Production from Composite Materials and New Metal Alloys, 21 Century, pp. 568-574, Russia, January, 2001, MSU, Moscow

Dmitriev, O.S., Kirillov, V.N., Mischenko, S.V. \& Dmitriev, S.O. (2009) Optimization of Curing Cycles Products of the Polymer Composite Materials on Base of Glutinous Prepregs. Polymer Science, Series D. Glues and Sealing Materials, Vol. 2, No. 4, (2009) pp. 223-229, ISSN 1995-4212

Dmitriev, O.S., Kirillov, V.N., Mischenko, S.V. \& Dmitriev, A.O. (2010) Computer-Measuring System for Research into Properties of Glutinous Prepregs and Calculation of Curing Cycles of the Polymer Composite Materials on Their Base. Polymer Science, Series D. Glues and Sealing Materials, Vol. 3, No. 1, (2010) pp. 20-25, ISSN 1995_4212

Frank, D., Laananen, D., Bogucki, G. et al (1991) Cure Optimization for Thin-Skinned Sandwich Structures, Proceedings of 36th International SAMPE Symposium, Vol. 36, pp. 999-1013, April, 1991, SAMPE

Kulichikhin, S.G. \& Astakhov, P.A. (1991) Optimization of forming cycles of fiberglass plastics based reokinetics analysis. Plast. mass., No. 11, (November 1991) pp. 36-38

Rosenberg, B.A. \& Enikolopyan, N.S. (1978) Problems of technological monolithic products of composite materials. Zhurn. Vsesoyuz. khim. obschestva, Vol. 23, No. 3, (March 1978) pp. 298-304

Stern, W.E. (1992) A Practical Method for the Cure Cycle Optimization of Epoxy Resins Via Differential Scanning Calorimetry, Proceedings of 37th International SAMPE Symposium, Vol. 37, pp. 231-239, March, 1992, SAMPE

Wu, H.T. \& Joseph, B. (1990). Knowledge Based Control of Autoclave Curing of Composites. SAMPE Journal, Vol. 26, No. 6, (June 1990) pp. 39-54 


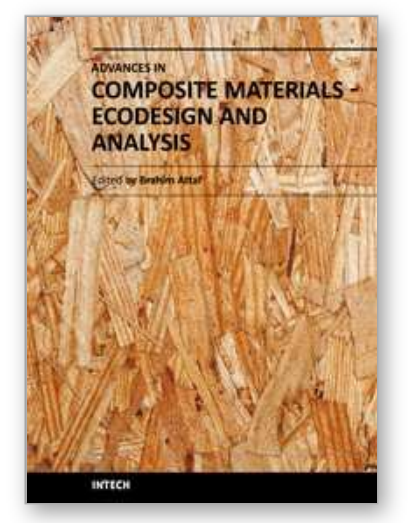

\author{
Advances in Composite Materials - Ecodesign and Analysis \\ Edited by Dr. Brahim Attaf
}

ISBN 978-953-307-150-3

Hard cover, 642 pages

Publisher InTech

Published online 16, March, 2011

Published in print edition March, 2011

By adopting the principles of sustainable design and cleaner production, this important book opens a new challenge in the world of composite materials and explores the achieved advancements of specialists in their respective areas of research and innovation. Contributions coming from both spaces of academia and industry were so diversified that the 28 chapters composing the book have been grouped into the following main parts: sustainable materials and ecodesign aspects, composite materials and curing processes, modelling and testing, strength of adhesive joints, characterization and thermal behaviour, all of which provides an invaluable overview of this fascinating subject area. Results achieved from theoretical, numerical and experimental investigations can help designers, manufacturers and suppliers involved with high-tech composite materials to boost competitiveness and innovation productivity.

\title{
How to reference
}

In order to correctly reference this scholarly work, feel free to copy and paste the following:

Oleg Dmitriev and Sergey Mischenko (2011). Optimization of Curing Cycles for Thick-wall Products of the Polymeric Composite Materials, Advances in Composite Materials - Ecodesign and Analysis, Dr. Brahim Attaf (Ed.), ISBN: 978-953-307-150-3, InTech, Available from: http://www.intechopen.com/books/advances-incomposite-materials-ecodesign-and-analysis/optimization-of-curing-cycles-for-thick-wall-products-of-thepolymeric-composite-materials

\section{INTECH}

open science | open minds

\author{
InTech Europe \\ University Campus STeP Ri \\ Slavka Krautzeka 83/A \\ 51000 Rijeka, Croatia \\ Phone: +385 (51) 770447 \\ Fax: +385 (51) 686166 \\ www.intechopen.com
}

\author{
InTech China \\ Unit 405, Office Block, Hotel Equatorial Shanghai \\ No.65, Yan An Road (West), Shanghai, 200040, China \\ 中国上海市延安西路65号上海国际贵都大饭店办公楼 405 单元 \\ Phone: +86-21-62489820 \\ Fax: $+86-21-62489821$
}


(C) 2011 The Author(s). Licensee IntechOpen. This chapter is distributed under the terms of the Creative Commons Attribution-NonCommercialShareAlike-3.0 License, which permits use, distribution and reproduction for non-commercial purposes, provided the original is properly cited and derivative works building on this content are distributed under the same license. 